\title{
Zoledronic acid inhibits osteoclastogenesis and bone resorptive function by suppressing RANKL-mediated NF-кB and JNK and their downstream signalling pathways
}

\author{
XIAO-LIN HUANG ${ }^{1,2}$, CHAO LIU $^{3}$, XUE-MEI SHI ${ }^{1}$, YU-TING CHENG ${ }^{2}$, \\ QIAN ZHOU ${ }^{2}$, JIAN-PING LI ${ }^{1}$ and JIAN LIAO ${ }^{2}$ \\ ${ }^{1}$ Stomatology Medical Center of Zhongshan People's Hospital, Zhongshan, Guangdong 528403; \\ ${ }^{2}$ School/Hospital of Stomatology, Guizhou Medical University, Guiyang, Guizhou 550004; \\ ${ }^{3}$ Department of Respiratory Disease, Zhongshan People's Hospital, Zhongshan, Guangdong 528403, P.R. China
}

Received May 19, 2021; Accepted November 23, 2021

DOI: $10.3892 / \mathrm{mmr} .2021 .12575$

\begin{abstract}
Targeting excessive osteoclast differentiation and activity is considered a valid therapeutic approach for osteoporosis. Zoledronic acid (ZOL) plays a pivotal role in regulating bone mineral density. However, the exact molecular mechanisms responsible for the inhibitory effects of ZOL on receptor activator of nuclear factor (NF)- $\mathrm{kB}$ ligand (RANKL)-induced osteoclast formation are not entirely clear. The present study aimed to investigate the role of ZOL in osteoclast differentiation and function, and to determine whether NF- $\kappa \mathrm{B}$ and mitogen-activated protein kinase, and their downstream signalling pathways, are involved in this process. RAW264.7 cells were cultured with RANKL for differentiation into osteoclasts, in either the presence or absence of ZOL. Osteoclast formation was observed by tartrate-resistant acid phosphatase staining and bone resorption pit assays using dentine slices. The expression of osteoclast-specific molecules was analysed using reverse transcription-quantitative polymerase chain reaction and western blotting assays to deduce the molecular mechanisms underlying the role of ZOL in osteoclastogenesis. The results showed that ZOL significantly attenuated osteoclastogenesis and bone resorptive capacity in vitro. ZOL also suppressed the activation of NF- $\mathrm{\kappa B}$ and the phosphorylation of c-Jun N-terminal kinase. Furthermore, it inhibited the expression of the downstream factors c-Jun, c-Fos and nuclear
\end{abstract}

Correspondence to: $\mathrm{Dr}$ Jian Liao, School/Hospital of Stomatology, Guizhou Medical University, 9 Beijing Road, Guiyang, Guizhou 550004, P.R. China

E-mail: liaojian@gmc.edu.cn

Dr Jian-Ping Li, Stomatology Medical Center of Zhongshan People's Hospital, 72 Hubin Road, Zhongshan, Guangdong 528403, P.R. China

E-mail: ljpdr126@126.com

Key words: osteoclast, bone resorption, zoledronic acid, osteoclastogenesis, osteoporosis, in vitro factor of activated $\mathrm{T}$ cells $\mathrm{c}$, thereby decreasing the expression of dendritic cell-specific transmembrane protein and other osteoclast-specific markers. In conclusion, ZOL may have therapeutic potential for osteoporosis.

\section{Introduction}

Under normal conditions, skeletal bone mass is maintained by the net balance between the number of bone-forming osteoblasts and bone-resorbing osteoclasts. If either of these bone cells undergo a change in activity, an imbalance in bone maintenance can occur. The dysregulation of bone remodelling can result in different types of skeletal diseases, such as osteoporosis and osteopetrosis (1). Osteoporosis, a global public health concern, is characterised by bone mass reduction and microarchitectural deterioration of bone, resulting in increased risk of fragility fractures (2). Osteoporosis-related fractures can cause disability, substantial pain and even death in patients with osteoporosis (3).

Osteoclasts are unique bone-resorbing and multinucleated giant cells derived from the fusion of hematopoietic precursor cells of the monocyte/macrophage lineage (4). This fusion is regulated by the fusion-related molecule dendritic cell-specific transmembrane protein (DC-STAMP). The failure of cell fusion can result in an increase in bone mass, as observed in osteopetrosis (5). In addition, osteoclast differentiation is mainly governed by two crucial cytokines: Macrophage colony-stimulating factor (M-CSF) and receptor activator of nuclear factor (NF) $-\kappa B$ ligand (RANKL). M-CSF is constitutively produced by mesenchymal cells in the bone marrow in response to osteotropic factors; it induces the differentiation of precursor cells into mature osteoclasts (6). RANKL is most abundantly expressed on the cell surfaces of bone marrow stromal cells, osteoblasts and osteocytes (7); it stimulates the commitment of osteoclast precursors to the osteoclastic phenotype by interacting with RANK, which is expressed in osteoclast precursors. The RANKL/RANK interaction leads to the recruitment of signalling adaptor molecule tumour necrosis factor receptor-associated factor 6 (TRAF6). This in turn triggers the activation of a series of downstream 
signalling pathways, activating all three mitogen-activated protein kinases (MAPKs) [c-Jun N-terminal kinase (JNK), extracellular signal-regulated kinase (ERK) and p38], as well as transcription factors such as NF- $\kappa B(8,9)$. NF- $\kappa B$ is a key pleiotropic transcription factor involved in the early stages of RANKL-induced osteoclast differentiation (10). Activation of the NF- $\kappa B$ signalling pathway promotes the phosphorylation and subsequent degradation of inhibitor of $\kappa \mathrm{B} \alpha(\mathrm{I} \kappa \mathrm{B} \alpha)$, followed by the phosphorylation and translocation of downstream p65 to the nucleus. In addition, MAPK activation results in the phosphorylation of c-Jun and its binding to c-Fos to form the essential activator protein-1 (AP-1) transcription factor, which ultimately evokes the induction and activation of nuclear factor of activated T cell cl (NFATc1), a well-known calcineurin- and calcium-regulating transcription factor that promotes osteoclast differentiation and function $(11,12)$. These transcription factors consequently regulate several target genes involved in bone matrix degradation, including tartrate resistant acid phosphatase (TRAP), calcitonin receptor (CTR) and RANK (13).

Bisphosphonates (BPs) are a class of antiresorptive drugs that have a high affinity with bone. BPs are widely used for the clinical treatment of osteoporosis, bone metastasis, multiple myeloma, breast cancer and Paget's disease, as they help prevent hypercalcemia, pain and pathological fractures (14-16). Furthermore, they have been revealed to prevent diabetes-induced bone loss, and can enhance bone density in diabetic animals (17). Zoledronic acid (ZOL) is the most widely used nitrogen-containing BP. ZOL inhibits the differentiation and apoptosis of osteoclasts (18-20). In previous years, ZOL has been applied in stomatology, and numerous studies have focused on the outcomes of dental extractions in patients using ZOL, either alone or in combination with steroids (21-23). Data are also available regarding the outcomes of placing dental implants in these patients (24). Numerous researchers have concluded that ZOL can reverse the negative effects of osteoporosis and improve the fixation and osseointegration of dental implants, for both local and systemic treatments, and in autologous bone grafts under osteoporotic conditions $(21,25)$. However, the utility of ZOL is currently limited, as it can give rise to several side effects, one of which is termed bisphosphonate-related osteonecrosis of the jaw (BRONJ) (26-28). BRONJ has been associated with studies in various fields, including general medicine, oncology, and dental, oral and maxillofacial surgical procedures. Despite this, researchers have reached a consensus that the benefits of BP treatment generally outweigh the risks $(21,29,30)$. Importantly, several oral health-related risk factors, including periodontal disease and oral infections, implants, poor oral health, tooth extractions and dentoalveolar surgery (before and during treatment) are considered to be triggers for BRONJ development. However, the exact molecular pathways involved in BRONJ pathogenesis require further investigation $(15,28,31)$. Additionally, the precise molecular mechanisms of ZOL in the treatment of osteoporosis remain unclear. Our previous study (32) reported that ZOL may inhibit NF- $\mathrm{KB}$ and JNK signalling by reducing the levels of p-IкB $\alpha, \mathrm{p}-\mathrm{p} 65$ and p-JNK in a time-dependent manner, but the evidence showing that ZOL blocks the RANKL-induced activation of the NF- $\kappa B$ and JNK signalling pathways is insufficient. The present study hypothesized that suppressing these signalling pathways may be an effective therapeutic approach for treating bone loss diseases, including osteoporosis. Therefore, the present study further investigated the mechanisms through which $\mathrm{ZOL}$ inhibits osteoclast differentiation and function in RAW264.7 cells. In particular, focus was given to its effects on the NF- $\mathrm{KB}$ and JNK/c-Jun/c-Fos/NFATc1/DC-STAMP signalling axis, to provide new strategies for the treatment of osteoporosis.

\section{Materials and methods}

Cells, reagents and antibodies. The $\alpha$-modification of Eagle's medium ( $\alpha$-MEM; cat. no. SH30265.01B) and fetal bovine serum (FBS; cat. no. 10099141C) were obtained from Gibco; Thermo Fisher Scientific, Inc. The RAW264.7 mouse macrophage cell line (osteoclast precursor; cat. no. TIB71) was purchased from the American Type Culture Collection. The recombinant murine soluble RANK ligand (sRANKL; cat. no. 315-11C) and macrophage colony-stimulating factor (M-CSF; cat. no. 315-02) were obtained from PeproTech, Inc. The TRAP staining kit (cat. no. 386 A) and ZOL (cat. no. SML0223-10MG) were purchased from Sigma-Aldrich; Merck KgaA. Specific antibodies against IкB $\alpha$ (cat. no. 4812), phospho-IкB $\alpha$ (p-IkBa; cat. no. 2859) (Ser32), p38 (cat. no. 8690), phospho-p38 (p-p38; cat. no. 4511) (Thr180/Tyr182), c-Jun N-terminal kinase (JNK; cat. no. 9252), phospho-JNK (p-JNK; cat. no. 4668) (Thr183/Tyr185), extracellular signal-regulated kinase 1/2 (ERK1/2; cat. no. 9102), phospho-ERK (p-ERK; cat. no. 4370) (Thr202/Tyr204), p65 (cat. no. 8242), phospho-p65 (p-p65, cat. no. 3033), c-Fos (cat. no. 2250), NFATc1 (cat. no. 8032) and c-Jun (cat. no. 9165) were obtained from Cell Signaling Technology, Inc. Specific antibodies against DC-STAMP (cat.no.MABF39-1) were purchased from Sigma-Aldrich; Merck KgaA. Specific antibodies against GAPDH (cat. no. AB-P-R 001) were obtained from Hangzhou Xianzhi Biotechnology Co., Ltd., and those against Lamin B1 (cat. no. bs-1840R) were obtained from Beijing Biosynthesis Biotechnology Co., Ltd. Horseradish peroxidase-conjugated goat anti-rabbit IgG secondary anti-body (cat. no. 014-090S) was obtained from PMK Bioprimacy Co., Ltd. ECL solution (cat. no. WBKLS0100) was obtained from EMD Millipore.

Osteoclastogenesis. Osteoclast differentiation was performed according to the method previously described by Chen et al (33). RAW264.7 cells were seeded in 96-well tissue culture plates with $\alpha$-MEM (10\% FBS and $1 \%$ penicillin-streptomycin) at a density of $1.5 \times 10^{3}$ cells/well and incubated at $37^{\circ} \mathrm{C}$ under $5 \% \mathrm{CO}_{2}$ and $95 \%$ humidity overnight. Cells were divided into four groups as follows: i) Vehicle; ii) RANKL-only; iii) RANKL + M-CSF and iv) RANKL + M-CSF + ZOL. Cells were cultured for 5 days. The conditioned medium was replaced with fresh $\alpha$-MEM every 2 days, and cells were then stained for TRAP at $37^{\circ} \mathrm{C}$ for $1 \mathrm{~h}$ using a TRAP staining kit according to the manufacturer's protocol. TRAP ${ }^{+}$cells with three or more nuclei were manually counted as mature multinucleated osteoclasts by bright field microscopy.

Detection of osteoclast bone resorption. To detect osteoclast bone resorption, sterile bovine bone slices (IDS Nordic) were placed in 96-well plates in triplicate. RAW264.7 cells were then placed onto the bovine bone slices in $\alpha$-MEM complete 
medium at a density of $1.5 \times 10^{3}$ cells/well, and the medium was replaced every $48 \mathrm{~h}$. After 10 days of induction, cells were brushed off the bone slices, and the resorption pits were observed using a scanning electron microscope (E-1010; Hitachi, Ltd.). Finally, the number of pits was quantified using ImageJ software 6.0 (National Institutes of Health) (34).

Reverse transcription-quantitative polymerase chain reaction $(R T-q P C R)$. RAW264.7 cells were seeded onto 6-well plates at a density of $1 \times 10^{5}$ cells/well and cultured in complete $\alpha$-MEM with $100 \mathrm{ng} / \mathrm{ml}$ RANKL. Cells were or were not treated with $1 \mu \mathrm{M} \mathrm{ZOL}$ at $37^{\circ} \mathrm{C}$ for $0,1,3$ and 5 days. Total RNA was extracted from the cells using TRIzol ${ }^{\circledR}$ reagent (Invitrogen; Thermo Fisher Scientific, Inc.). cDNA was synthesised from $1 \mathrm{mg}$ of total RNA using the PrimeScript RT reagent kit (Takara Bio, Inc.) according to the manufacturer's protocol and stored at $-70^{\circ} \mathrm{C}$ until further use. qPCR was performed using the SYBR ${ }^{\circledR}$ Premix Ex Taq ${ }^{\mathrm{TM}}$ kit (TaKaRa Bio, Inc.). Thermocycling conditions were as follows: Initial denaturation for 2 min at $95^{\circ} \mathrm{C}$, followed by 40 cycles of denaturation at $95^{\circ} \mathrm{C}$ for $15 \mathrm{sec}$ and amplification at $60^{\circ} \mathrm{C}$ for $1 \mathrm{~min}$ and final extension for $15 \mathrm{sec}$ at $95^{\circ} \mathrm{C}, 15 \mathrm{sec}$ at $60^{\circ} \mathrm{C}$ and $15 \mathrm{sec}$ at $95^{\circ} \mathrm{C}$. The $2^{-\Delta \Delta \mathrm{Cq}}$ method was used to calculate the relative mRNA expression, and all reactions were performed in triplicate (35). GAPDH was used as a quantitative control for the expression levels of each gene in all experimental groups (33). Data is expressed as fold-change relative to the control. The refseq of NFATc1, c-fos, DC-STAMP, TRAP, RANK, CTR, and GAPDH were as follows: TRAP, 001102405.1; DS-STAMP, 029422.4; RANK, AF019046.1; NFATc1, 016791.4; CTR, 001355192.1; c-fos, 010234.3; and GAPDH, 001289726.1. The primer sequences of these osteoclast-specific markers and GAPDH are listed in Table I.

Western blot analysis. Total protein was extracted from cells using RIPA buffer containing $150 \mathrm{mM} \mathrm{NaCl}, 5 \mathrm{mM}$ EDTA, $50 \mathrm{mM}$ Tris- $\mathrm{HCl}, 1 \mathrm{mM}$ sodium fluoride, $1 \mathrm{mM}$ sodium vanadate, $1 \%$ Triton $\mathrm{X}-100,1 \%$ phosphatase and $1 \%$ protease inhibitors. The protein concentrations were quantified using the bicinchoninic acid method. Next, total protein (30 $\mu \mathrm{g}$ per lane) was subjected to $10 \%$ SDS-PAGE and transferred to PVDF membranes. Following transfer, membranes were blocked with 5\% non-fat milk in Tris-buffered saline containing $0.1 \%$ Tween-20 at room temperature for $2 \mathrm{~h}$. After incubation with indicated primary antibodies [p-ERK $(1: 1,000)$, ERK $(1: 1,000)$, p-p38 (1:1,000), p38 (1:1,000), p-IкB $\alpha(1: 1,000)$, IкB $\alpha,(1: 1,000)$, p-JNK (1:1,000), JNK (1:1,000), p-p65 (1:1,000), p65 (1:1,000), NFATc1 (1:1,000), c-Fos (1:600), DC-STAMP (1:800), c-Jun $(1: 1,000)$, Lamin B1 $(1: 1,000)$ and GAPDH $(1: 1,000)]$ overnight at $4^{\circ} \mathrm{C}$, the membranes were washed and then incubated with horseradish peroxidase-conjugated secondary antibodies diluted at 1:10,000 for $1 \mathrm{~h}$ at room temperature. ECL was used to develop a fluorescent signal. Antibody reactivity was detected using the Gene Gnome Imaging System (Syngene Europe) and band densities were quantified using ImageJ software 6.0 (National Institutes of Health). Only representative blots are shown.

NFATcl reporter assay. To examine whether ZOL inhibited NFATc1 activation, RAW264.7 cells stably transfected with a luciferase reporter construct NFATc1-Luc were seeded
Table I. Sequences of primers used in quantitative PCR.

Primer Gene sequence (5'-3')

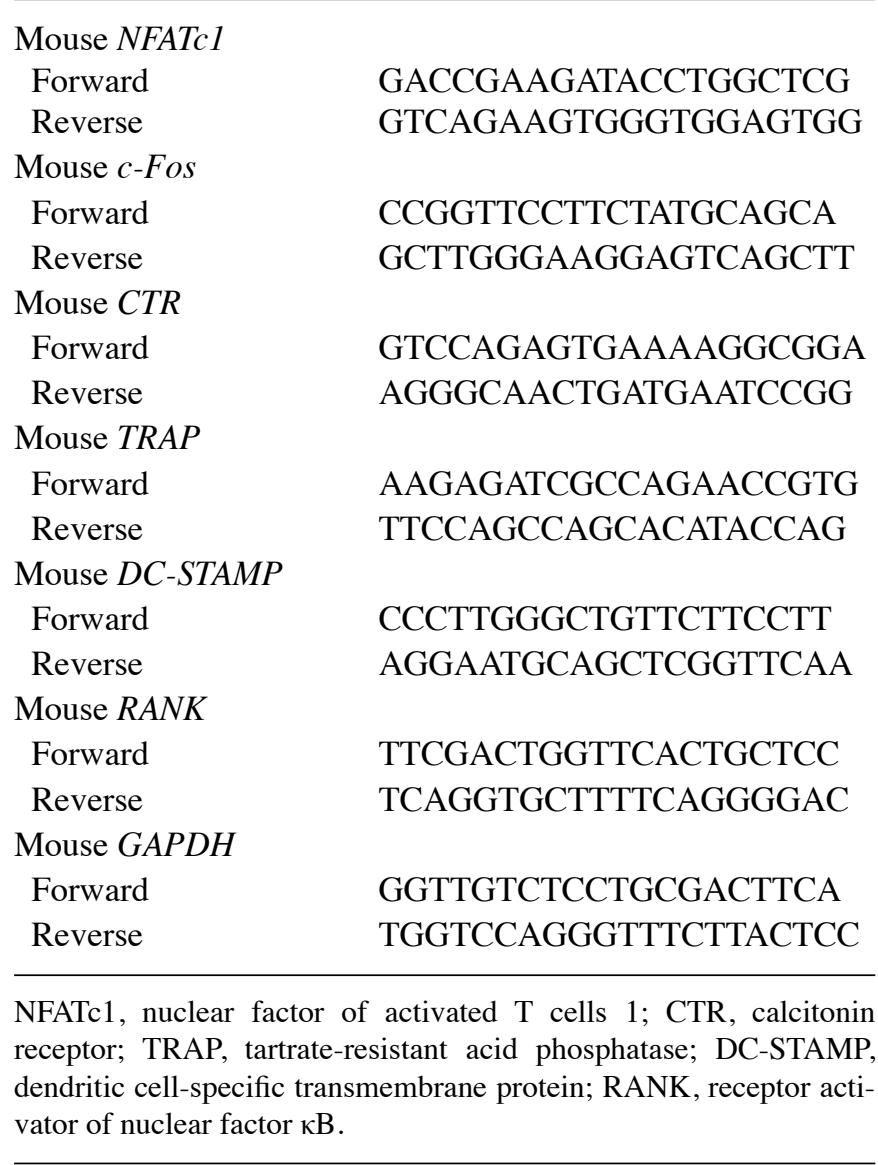

onto 96-well plates at a density of $1 \times 10^{4}$ cells/well. These cells were pre-treated with $1 \mu \mathrm{M}$ ZOL for $1 \mathrm{~h}$, and then incubated in the absence or presence of $100 \mathrm{ng} / \mathrm{ml}$ RANKL at $37^{\circ} \mathrm{C}$ for $8 \mathrm{~h}$. At the end of the treatments, cells were lysed and luciferase activity was measured using Luciferase Assay System according to the manufacturer's protocol (Promega Corporation).

Statistical analysis. All data were collected from at least three independent experiments. Values are expressed as the mean \pm standard deviation. An unpaired Student's t-test was used for comparisons between two groups, and one-way ANOVA followed by Tukey's post-hoc test was used for multiple comparisons, assessed via GraphPad Prism 6.0 (GraphPad Software, Inc.). $\mathrm{P}<0.05$ was considered to indicate a statistically significant difference.

\section{Results}

ZOL inhibits RANKL-mediated osteoclastogenesis and bone resorption. Increasing evidence has suggested that RANKL and M-CSF are sufficient and necessary for the formation and differentiation of osteoclasts (36-38). Our previous study revealed that ZOL had no cytotoxic effects on RAW264.7 cells at the concentrations used in the present study (32). The present study confirmed that RAW264.7 cells differentiated into 


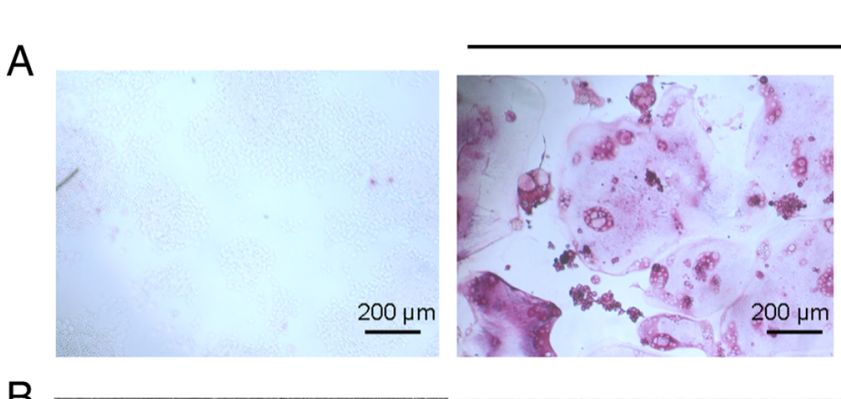

RANKL $(100 \mathrm{ng} / \mathrm{ml})$
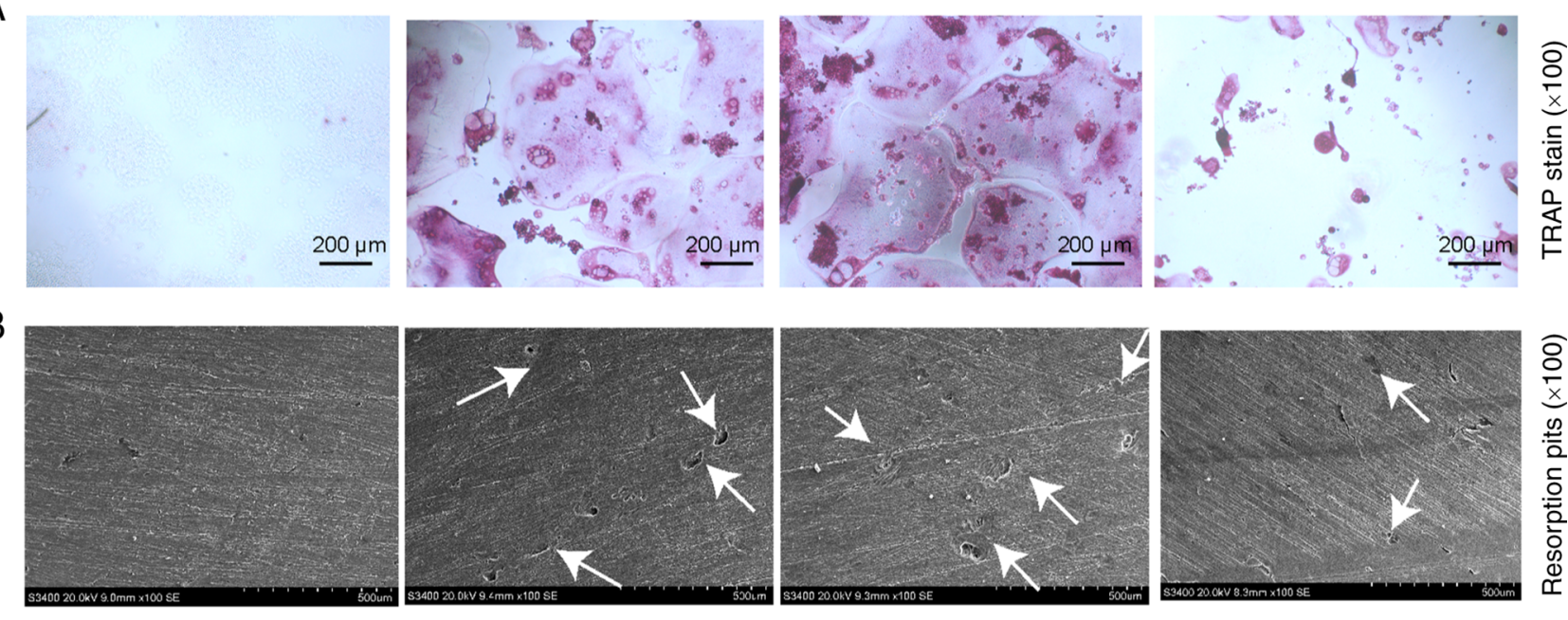

$\mathrm{M}-\mathrm{CSF}(50 \mathrm{ng} / \mathrm{ml})$

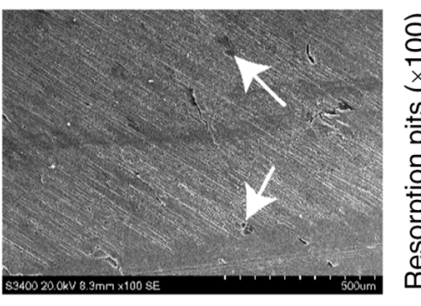

$\mathrm{ZOL}(1 \mu \mathrm{M})$
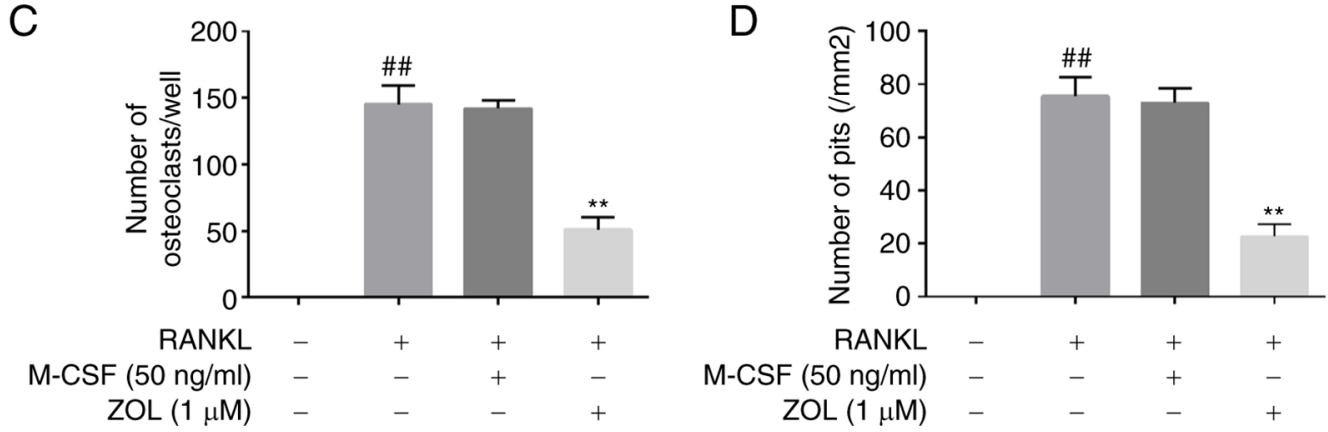

Figure 1. Inhibition of RANKL-induced osteoclast formation and bone resorption in RAW264.7 cells by ZOL. Images showing (A) TRAP staining and (B) bone resorption pits (indicated by white arrows). Scale bars: (A) $200 \mu \mathrm{m}$ and (B) $500 \mu \mathrm{m}$. (C) RANKL-induced formation of TRAP ${ }^{+}$multinucleated osteoclasts. (D) Determination of number of resorption pits. ${ }^{\# \#} \mathrm{P}<0.01$ vs. vehicle group and ${ }^{* *} \mathrm{P}<0.01$ vs. RANKL-only group. ZOL, zoledronic acid; TRAP, tartrate-resistant acid phosphatase; M-CSF, macrophage colony-stimulating factor; RANKL, receptor activator of nuclear factor- $\kappa \mathrm{B}$ ligand.

$\mathrm{TRAP}^{+}$multinucleated osteoclasts (Fig. 1A) and subsequently displayed enhanced bone resorptive function (Fig. 1B) in the presence of RANKL. However, no significant difference was observed between the RANKL and RANKL + M-CSF groups. Therefore, RAW264.7 cells were cultured in the presence of $100 \mathrm{ng} / \mathrm{ml}$ RANKL (with no M-CSF) for subsequent experiments. The effects of ZOL on osteoclast differentiation and resorption pit formation were then investigated. Pre-treatment with ZOL strongly inhibited the RANKL-induced formation of $\mathrm{TRAP}^{+}$multinucleated osteoclasts (Fig. 1A) and bone-resorbing pits (Fig. 1B). The numbers of osteoclasts (Fig. 1C) and resorption pits (Fig. 1D) were both significantly reduced following incubation with $1 \mu \mathrm{M}$ ZOL. These findings convincingly demonstrated that ZOL inhibits the fusion of osteoclast precursors and the bone-resorbing activity of mature osteoclasts.

ZOL inhibits the expression of osteoclast-specific markers. The effects of ZOL on osteoclast-specific markers were further explored by analysing the mRNA expression levels of $R A N K, T R A P$ and $C T R$ in the absence or presence of ZOL. The stimulation of RAW264.7 cells with RANKL markedly induced the expression of these osteoclast marker genes. By contrast, treatment with ZOL after 3 days of RANKL stimulation markedly suppressed the mRNA expression levels of
RANK, TRAP and CTR (Fig. 2). These results are consistent with the previous finding that ZOL inhibits osteoclastogenesis and bone resorption.

Effect of ZOL on RANKL-induced $N F-\kappa B$ activation. The $\mathrm{NF}-\kappa \mathrm{B}$ pathway plays a vital role in RANKL-induced osteoclastogenesis. To investigate the molecular mechanism by which ZOL suppresses the proteins associated with osteoclast differentiation, the present study first focused on the effect of ZOL on $N F-\kappa B$ activation by analysing the phosphorylation of $\mathrm{I} \kappa \mathrm{B} \alpha$ (the inhibitor of $\mathrm{NF}-\kappa \mathrm{B}$ ) and the phosphorylation and translocation of p65, all of which are critical steps in $\mathrm{NF}-\kappa \mathrm{B}$ activation. The rapid phosphorylation of $\mathrm{I} \kappa \mathrm{B} \alpha$ and the p65 subunit was detected in RAW264.7 cells treated with RANKL, indicating that the NF- $\kappa \mathrm{B}$ pathway was activated. However, ZOL downregulated the RANKL-induced phosphorylation of $\mathrm{I} \kappa \mathrm{B} \alpha$, while upregulating the level of non-phosphorylated I $\mathrm{B} \alpha$. Similarly, RANKL-induced phosphorylation of p65 was attenuated by ZOL in a dose-dependent manner (Fig. 3A-D). To further confirm this finding, the nuclear translocation of p65 was assessed by western blotting of cytosolic and nuclear extracts, which revealed that RANKL treatment increased p65 levels in nuclear extracts. By contrast, treatment with ZOL, followed by stimulation with RANKL, inhibited the nuclear 

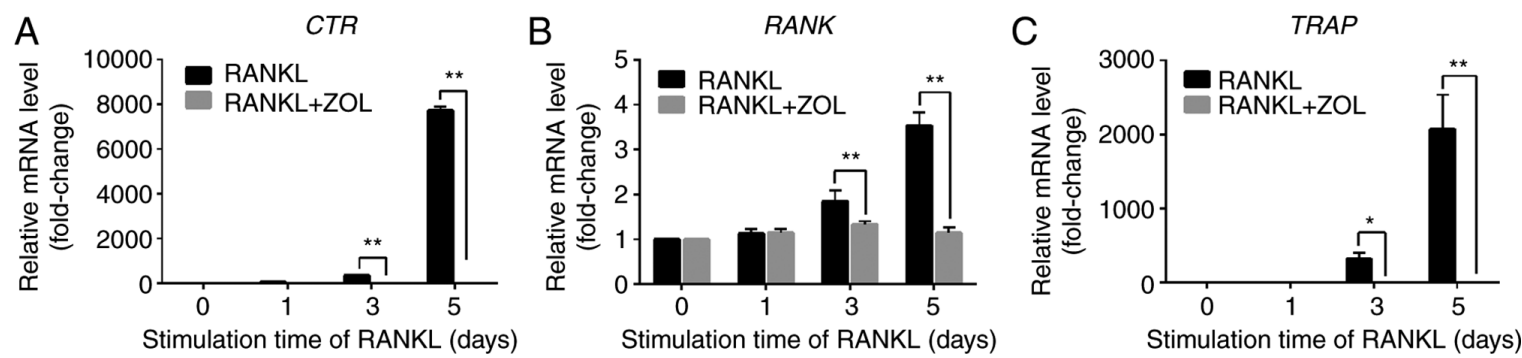

Figure 2. Inhibition of RANKL-induced expression of osteoclastic marker genes by ZOL. (A) CTR, (B) RANK and (C) TRAP. ${ }^{*} \mathrm{P}<0.05$, ${ }^{* *} \mathrm{P}<0.01$ vs. RANKL-only group. ZOL, zoledronic acid; RANKL, receptor activator of nuclear- $\kappa \mathrm{B}$ ligand; CTR, calcitonin receptor; TRAP, tartrate-resistant acid phosphatase.

A

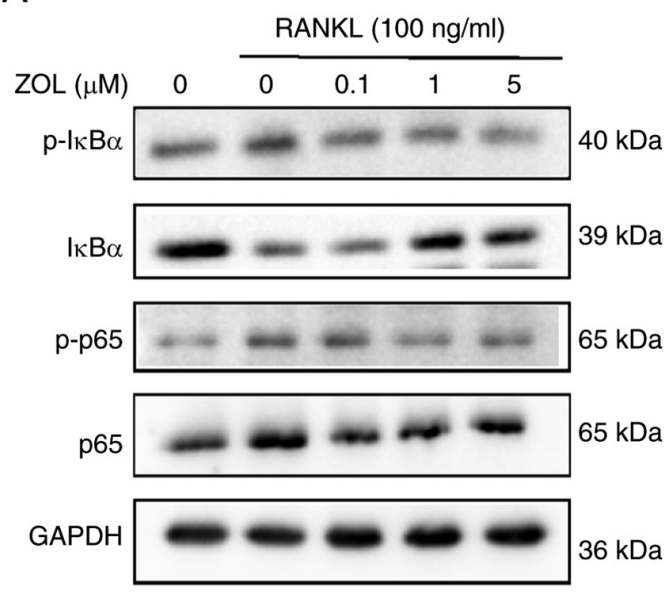

E

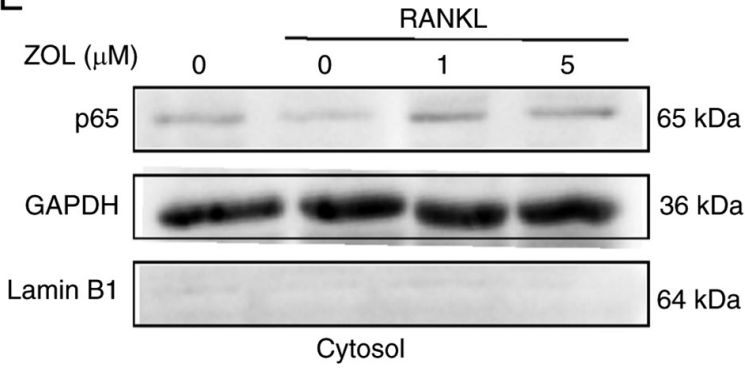

G

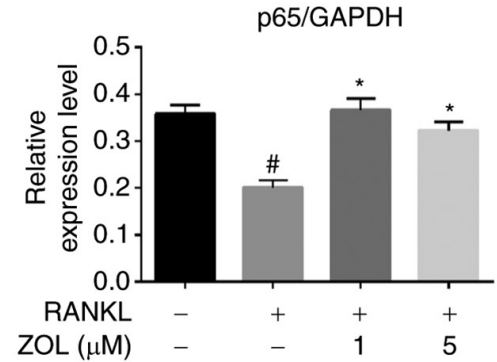

B

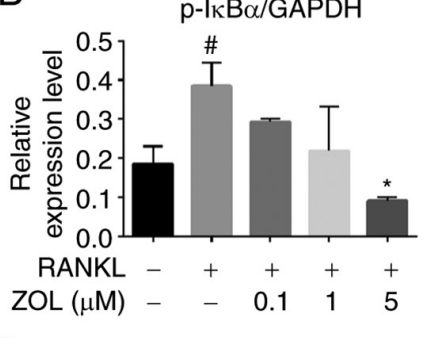

$\mathrm{D}$

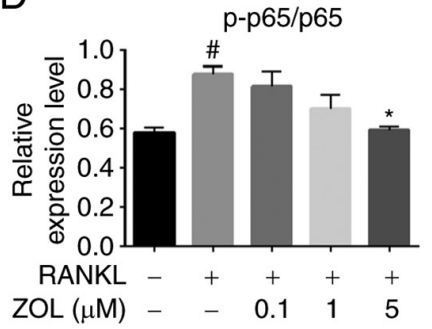

C

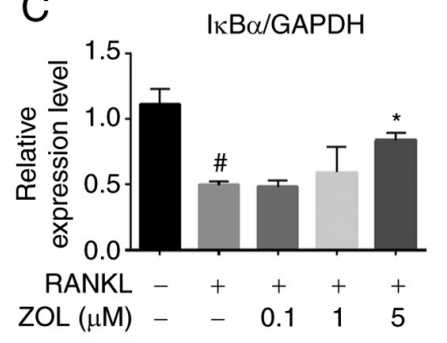

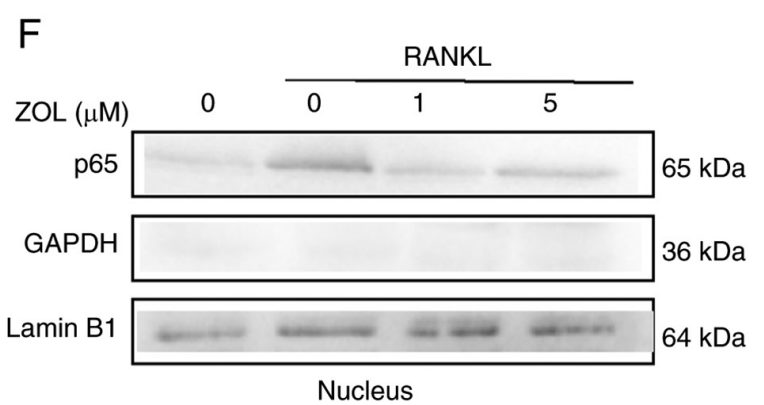

Nucleus

$\mathrm{H}$

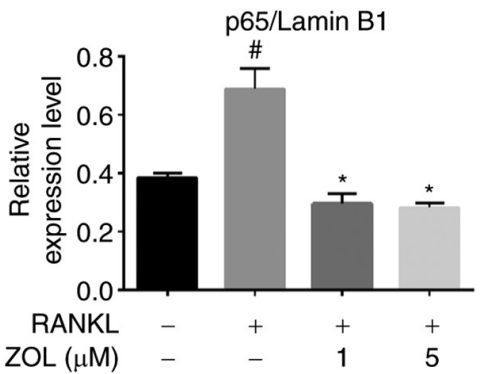

Figure 3. Inhibition of RANKL-induced NF- $\mathrm{kB}$ activity by ZOL. (A) RAW264.7 osteoclast precursors treated with RANKL for 30 min following pre-treatment

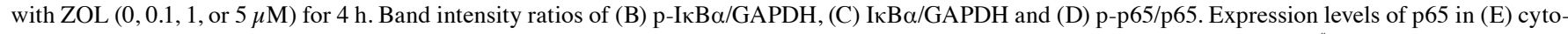
plasmic and (F) nuclear extracts, as analysed via western blotting. Band intensity ratios of $(\mathrm{G}) \mathrm{p} 65 / \mathrm{GAPDH}$ and $(\mathrm{H}) \mathrm{p} 65 / \mathrm{Lamin} \mathrm{B} 1 .{ }^{. \prime} \mathrm{P}<0.05$ vs. vehicle group and " $\mathrm{P}<0.05$ vs. RANKL-only group. ZOL, zoledronic acid; RANKL, receptor activator of nuclear factor- $\kappa \mathrm{B}$ ligand; $\mathrm{p}$-I $\mathrm{B} \alpha$, phosphorylated inhibitor of $\kappa \mathrm{B} \alpha$.

translocation of p65 (Fig. 3E-H). These results suggested that the inhibitory effects of ZOL on RANKL-induced osteoclast differentiation and bone resorption may occur in part due to inhibition of the NF- $\mathrm{kB}$ pathway.
ZOL inhibits RANKL-induced JNK phosphorylation. MAPKs are located downstream of TRAF6 signalling complexes and play a significant role in RANKL-mediated osteoclast differentiation (39). Therefore, the effects of ZOL on MAPK 
A

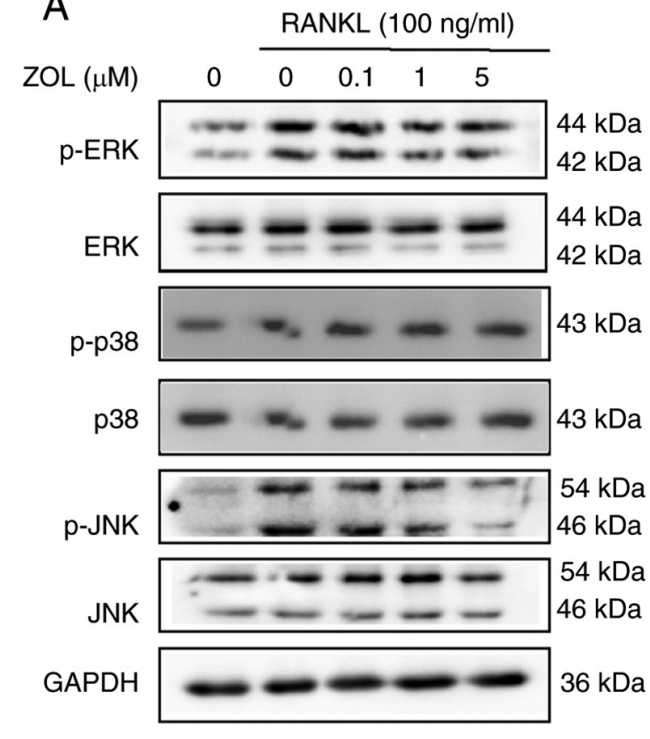

B

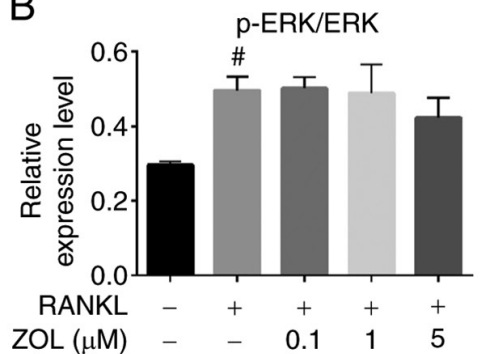

D

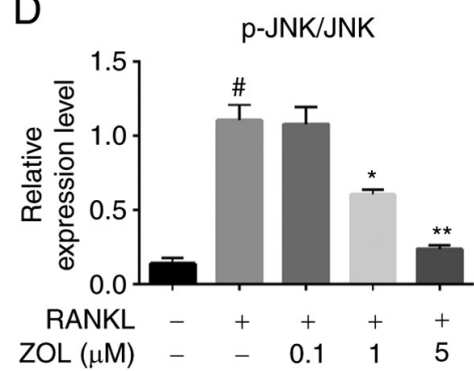

C

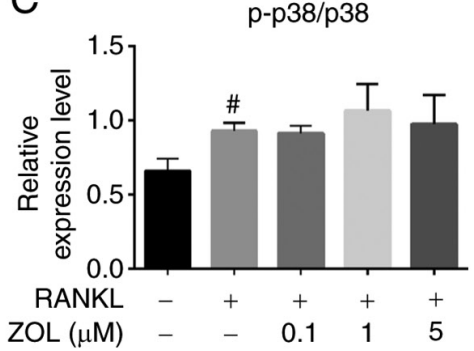

$\mathrm{ZOL}(\mu \mathrm{M}) \quad-\quad-0.1 \quad 1 \quad 5$

Figure 4. Suppression of RANKL-induced JNK signalling by ZOL. (A) RAW264.7 osteoclast precursors treated with RANKL for 30 min following pretreatment with ZOL $(0,0.1,1$, or $5 \mu \mathrm{M})$ for $4 \mathrm{~h}$. Band intensity ratios of (B) p-ERK/ERK, (C) p-p38/p38 and (D) p-JNK/JNK. "P<0.05 vs. vehicle group; ${ }^{*} \mathrm{P}<0.05$ and ${ }^{* *} \mathrm{P}<0.01$ vs. RANKL-only group. ZOL, zoledronic acid; RANKL, receptor activator of nuclear factor- $\kappa \mathrm{B}$ ligand; JNK, c-Jun N-terminal kinase; ERK1/2, extracellular regulated protein kinases.

family proteins were examined. Treating RAW264.7 cells with RANKL increased the phosphorylation of p38, JNK and ERK. By contrast, ZOL treatment downregulated JNK phosphorylation in a dose-dependent manner. However, the phosphorylation of the p38 and ERK proteins was not significantly affected by ZOL (Fig. 4). These results suggested that ZOL suppresses RANKL-induced JNK phosphorylation during osteoclast differentiation.

ZOL suppresses RANKL-induced downstream expression of c-Jun, $c$-Fos and NFATcl. As ZOL can suppress RANKL-induced activation of JNK and NF- $\kappa$ B during osteoclastogenesis, the ability of ZOL to inhibit the downstream expression of c-Jun, NFATc1 and c-Fos was further explored. The expression of c-Jun, which is downstream of JNK, was increased in RANKL-stimulated RAW264.7 cells and was reduced significantly by exposure to ZOL. Consistent with aforementioned results, the mRNA and protein expression levels of c-Fos and NFATc1 both increased from the first day of RANKL treatment. However, ZOL treatment significantly reduced the RANKL-induced $\mathrm{mRNA}$ and protein expression of these two transcription factors (Fig. 5A-J). Furthermore, a luciferase reporter assay revealed that the transcriptional activity of NFATc1 signalling was also significantly inhibited by ZOL treatment (Fig. 5K). Collectively, these results suggested that ZOL targets the upstream kinase to inhibit the expression of downstream functional transcription factors.

ZOL negatively regulates $R A N K L$-induced expression of fusion-related molecules DC-STAMP. Cell-cell fusion is crucial for the formation of osteoclasts, and fusion-related molecule DC-STAMP is involved in this process (38). To determine whether the suppressive effect of ZOL on osteoclast differentiation and bone resorption arose from the inhibition of cell-cell fusion, DC-STAMP expression was examined using qPCR and western blot analyses. The results indicated that RANKL-induced protein expression of DC-STAMP was significantly inhibited by ZOL compared with the RANKL control (Fig. 6A and B). ZOL treatment also suppressed the RANKL-induced mRNA expression of DC-STAMP (Fig. 6C). These results indicated that the inhibitory effect of ZOL on the RANKL-induced expression of DC-STAMP may also suppress RANKL-induced osteoclast differentiation and bone resorption.

\section{Discussion}

Osteoclasts are known to mediate physiological bone remodelling during tooth eruption, bone growth and fracture healing $(40,41)$. Notably, to date, no endogenous factors other than RANKL have been found to induce osteoclast formation without RANKL participation. Additionally, M-CSF is vital for providing osteoclast precursor cells with proliferation and survival signals, and increasing RANK expression, which is a prerequisite for the differentiation and function of osteoclasts (3). However, no significant difference was here observed between the RANKL and RANKL + M-CSF groups during osteoclast differentiation in RAW264.7 cells. Therefore, in the follow-up study, the RANKL-induced osteoclast differentiation platform was adopted to examine the effect of ZOL on osteoclast formation in RAW264.7 cells. The results of the present study revealed that ZOL significantly inhibited RANKL-induced osteoclastogenesis without any cytotoxic effects. Bone resorption is known to occur in conjunction with osteoclastogenesis. The present study confirmed that ZOL significantly suppressed both mature osteoclast formation and bone resorptive function. These findings partly explain the effectiveness of ZOL in treating bone-destroying skeletal diseases.

The role of NF- $\mathrm{NB}$ in osteoclastogenesis and bone homeostasis has been widely investigated (42). NF- $\kappa \mathrm{B}-\mathrm{knockout} \mathrm{mice}$ 
A

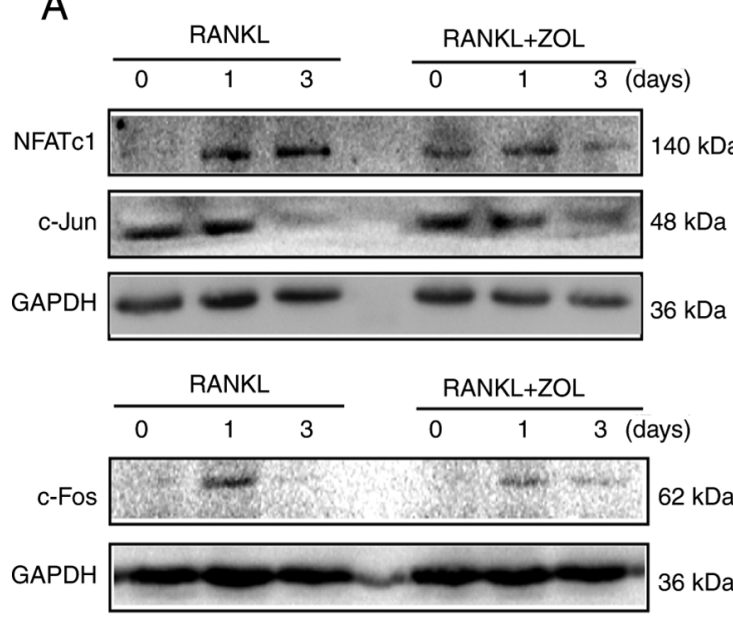

E
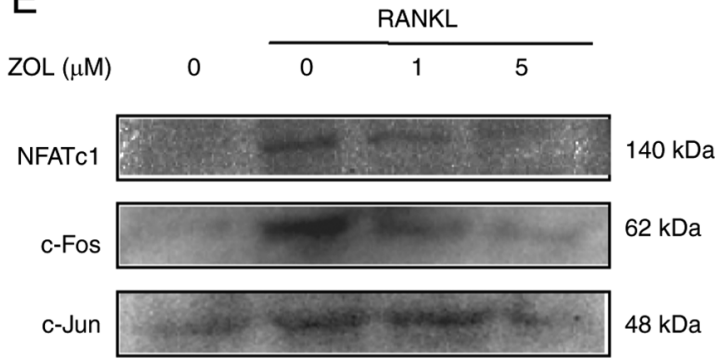

GAPDH

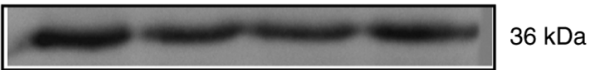

B

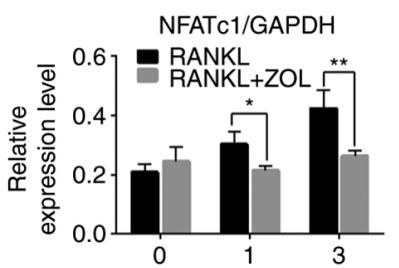

Stimulation time of RANKL (days)
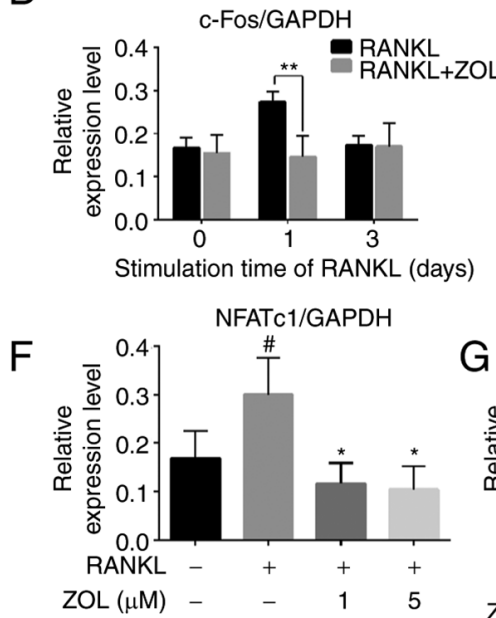

c-Jun/GAPDH

$\mathrm{H}$

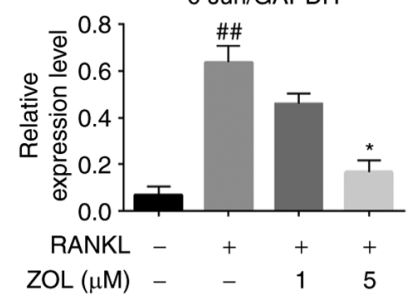

C C-Jun/GAPDH

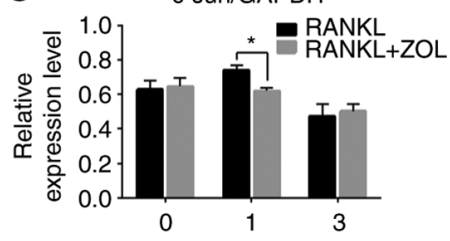

Stimulation time of RANKL (days)
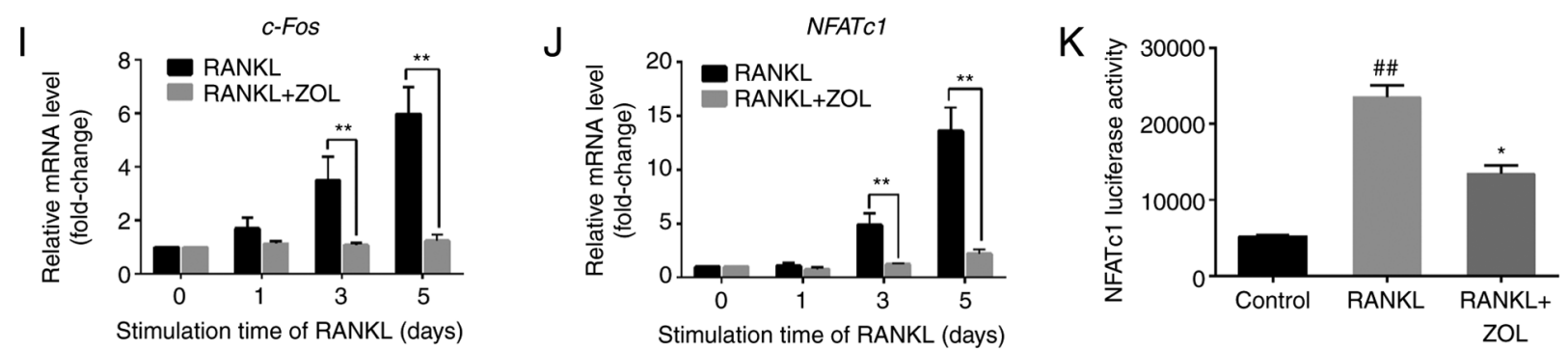

Figure 5. Suppression of RANKL-induced expression of downstream c-Jun, c-Fos and NFATc1 by ZOL. (A) RAW264.7 cells incubated in 100 ng/ml RANKL with or without $1 \mu \mathrm{M}$ ZOL for 0,1 or 3 days. The protein expression level was detected by western blotting. Only representative blots are shown (c-Fos is from a different membrane to the other bands). Band intensity ratios of (B) NFATc1/GAPDH, (C) c-Jun/GAPDH and (D) c-Fos/GAPDH. (E) RAW264.7 cells cultured in $100 \mathrm{ng} / \mathrm{ml}$ RANKL with 0,1 or $5 \mu \mathrm{M}$ ZOL for 1 day. Band intensity ratios of (F) NFATc1/GAPDH, (G) c-Fos/GAPDH and (H) c-Jun/GAPDH. Expression of osteoclast-specific genes (I) $c$-Fos and (J) NFATcl, as detected via quantitative PCR. (K) ZOL inhibits NFATc1 transcriptional activity, as determined by the Promega Luciferase Assay System. ${ }^{\#} \mathrm{P}<0.05$ and ${ }^{\# \#} \mathrm{P}<0.01$ vs. vehicle group; ${ }^{*} \mathrm{P}<0.05$ and ${ }^{* *} \mathrm{P}<0.01$ vs. RANKL-only group. ZOL, zoledronic acid; RANKL, receptor activator of nuclear factor- $\kappa \mathrm{B}$ ligand; NFATc1, nuclear factor of activated $\mathrm{T}$ cells 1 .

have shown defects in osteoclast differentiation and severe osteopetrosis (39). In addition, in an unstimulated state, $\mathrm{NF}-\kappa \mathrm{B}$ exists in the cytoplasm as a complex with $\mathrm{I} \kappa \mathrm{B}$, an inhibitory protein. When the NF- $\kappa \mathrm{B}$ signalling pathway is activated by RANKL, I $\kappa$ B is phosphorylated and degraded. The p65/p50 heterodimer then translocates to the nucleus, thereby activating the transcription of osteoclastogenesis-related genes. In the present study, western blot analysis revealed that the suppressive effect of ZOL on osteoclastogenesis was dependent on the $N F-\kappa B$ signalling pathway. $N F-\kappa B$ signalling in RAW264.7 cells was activated by RANKL treatment but was suppressed by ZOL treatment. In particular, pre-treatment with ZOL markedly attenuated RANKL-induced NF- $\kappa \mathrm{B}$ activation by inhibiting I $\kappa \mathrm{B} \alpha$ degradation and the phosphorylation and nuclear translocation of p65 in RAW264.7 cells. These results suggested that ZOL inhibits RANKL-induced osteoclast differentiation by blocking $\mathrm{NF}-\kappa \mathrm{B}$ activation.

Downstream of RANKL/RANK signalling, JNK, p38 and ERK have been implicated as prominent regulators of various cellular responses, such as apoptosis, differentiation and cell proliferation $(43,44)$. Specifically, RANKL-activated MAPKs play vital roles in osteoclastogenesis. Thus, they are essential 
A

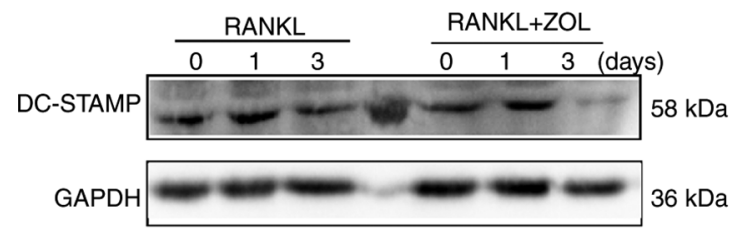

B

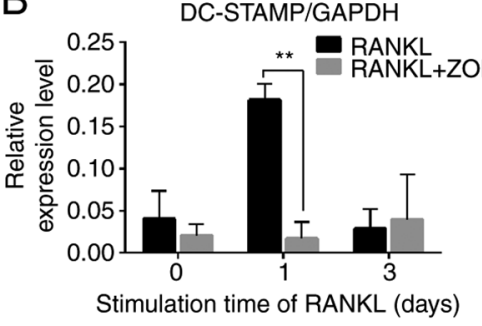

C

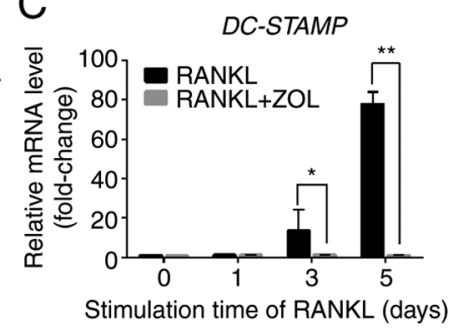

Figure 6. Suppression of expression level of DC-STAMP by ZOL. (A) RAW264.7 cells cultured in $100 \mathrm{ng} / \mathrm{ml}$ RANKL with or without $1 \mu$ M ZOL for 0 , 1 or 3 days. (B) Band intensity ratio of DC-STAMP/GAPDH. (C) RAW264.7 cells cultured in RANKL and treated with or without $1 \mu$ M ZOL for $0,1,3$ or 5 days. ${ }^{*} \mathrm{P}<0.05$ and ${ }^{* *} \mathrm{P}<0.01$ vs. RANKL-only group. DC-STAMP, dendritic cell-specific transmembrane protein; ZOL, zoledronic acid; RANKL, receptor activator of nuclear factor- $\kappa \mathrm{B}$ ligand.

molecular targets for therapeutic applications in inflammatory bone diseases $(33,45,46)$. The inhibition of p38, ERK and JNK can disturb RANKL-induced osteoclastogenesis $(11,47,48)$. In the present study, the effects of ZOL on the MAPK signalling pathway were investigated, demonstrating that ZOL significantly inhibited the phosphorylation of JNK, but did not significantly inhibit ERK or p38 signalling in RAW264.7 cells. The significance of JNK signalling in osteoclastogenesis has been previously reported. The activated JNK phosphorylates c-Jun and c-Fos bind to form heterodimers of AP-1, a crucial transcription factor during osteoclast differentiation (49). NFATc1 is another transcription factor that plays a crucial role in osteoclast formation by upregulating several genes responsible for osteoclast acidification, migration and adhesion, and for the degradation of inorganic and organic bone matrix (50). NFATc1-deficient embryonic stem cells cannot form mature osteoclasts via RANKL exposure, and the overexpression of NFATc1 in osteoclast precursors induces osteoclast differentiation $(39,51)$. The results presented in the current study revealed that ZOL treatment not only suppressed JNK phosphorylation, but also downregulated c-Jun levels. It was also identified that ZOL treatment reduced RANKL-induced NFATc1 activation in a luciferase activity assay, which is consistent with the evidence demonstrating that the stimulation of RAW264.7 cells with RANKL significantly upregulated the mRNA and protein expression levels of c-Fos and NFATc1, and this effect could be significantly suppressed by ZOL treatment. Additionally, the present study demonstrated that ZOL suppressed the expression of specific phenotypic markers, including TRAP, RANK and CTR. This indicated that the JNK/c-Jun/c-Fos/NFATc1 signalling axis may be involved in the inhibitory effects of ZOL on osteoclast differentiation.

In osteoclast precursors, RAW264.7 cells first proliferate and are then induced to become $\mathrm{TRAP}^{+}$mononuclear cells, which are termed preosteoclasts. These preosteoclasts then fuse together under the regulation of the fusion-related molecule DC-STAMP to form mature osteoclasts, and cell-cell fusion determines osteoclast size and initiates osteoclast bone resorptive activity (52). Without fusion-related molecules, RAW264.7 cells will only proliferate. After the effects of these molecules, RAW264.7 cells were found to preferentially differentiate into osteoclasts instead of proliferating. An anti-DC-STAMP monoclonal antibody has been reported to strongly inhibit osteoclast formation in vitro (53). Furthermore, DC-STAMP-deficient cells cannot differentiate into multinucleated osteoclasts and suffer from impaired bone resorptive function (54). As the expression of DC-STAMP is induced by RANKL/RANK signalling (38), it was hypothesized in the present study that DC-STAMP may also be involved in the inhibitory effects of ZOL on osteoclast differentiation. The data of the present study revealed that $\mathrm{ZOL}$ treatment reduced the expression of DC-STAMP at both the protein and mRNA levels. Therefore, ZOL may suppress both RANKL-mediated osteoclastogenesis and bone resorptive capacity by downregulating the expression of DC-STAMP.

As the RANKL/RANK pathway plays a key role in the pathological processes that induce bone loss, RANKL-targeted therapy is a valid approach for treating osteoporosis. In addition to the results of the present study, it has been previously demonstrated that the inhibiting effect of ZOL is involved in the RANKL/RANK pathway $(19,55)$. Denosumab, another effective medication for osteoporosis, is a fully human monoclonal anti-RANKL antibody that has high affinity and specificity for RANKL. However, whether denosumab is a valid alternative for patients unable to receive standard adjuvant i.v. ZOL remains controversial. Lee et al (56) conducted a large population-based cohort study using claims data (2010-15) from two large US commercial insurance databases to explore ocular outcomes in patients with osteoporosis in whom treatment was initiated with either ZOL or denosumab. It was identified that initiation of denosumab decreased osteoclast activity and exerted a prolonged effect on calcium metabolism, leading to decreased calcium deposition in the lens and lower likelihood of necessary cataract surgery compared with that of ZOL, but that the risk of age-related macular degeneration was similar between the two drugs. Kondo et al (57) suggested that sequential therapy using ZOL could suppress decreases in bone mineral density and increase of bone turnover marker if the period of denosumab administration was $<3$ years. Mori et al (58) used a simulation model to evaluate the effectiveness and cost of two treatment strategies. It was found that annual i.v. ZOL was more economical than biannual subcutaneous denosumab followed by weekly oral alendronate for 3 years. Notably, both ZOL and denosumab have been associated with BRONJ. Ikesue et al (59) evaluated the association between clinical characteristics and development of BRONJ in patients who underwent dental examinations before the initiation of treatment with denosumab or ZOL. The data suggested that BRONJ caused by denosumab resolves faster than that caused by ZOL. It was also reported that switching 


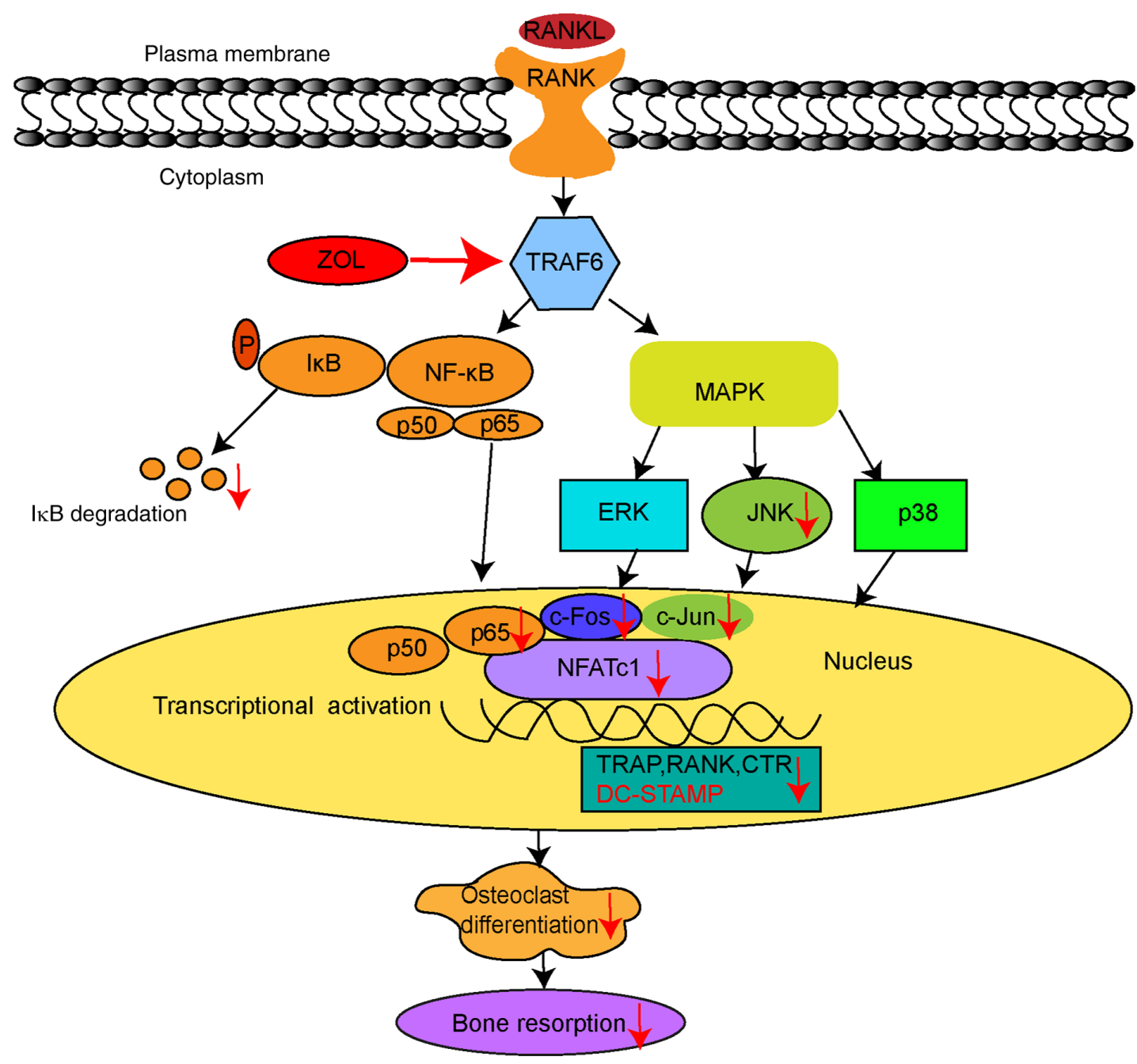

Figure 7. Schematic model for the action of ZOL treatment on the RANKL/RANK signalling pathway during osteoclast differentiation and bone resorption. The figure summarizes the results of the present study. Vertical arrows indicate either downregulation or inhibition of osteoclasts. TRAF6, tumour necrosis factor-associated factor 6; ZOL, zoledronic acid; JNK, c-Jun N-terminal kinase; ERK1/2, extracellular regulated protein kinases; TRAP, tartrate-resistant acid phosphatase; NF- $\mathrm{kB}$, nuclear factor- $\mathrm{\kappa}$; DC-STAMP, dendritic cell-specific transmembrane protein; CTR, calcitonin receptor; NFATc1, nuclear factor of activated T cells 1 ; RANKL, receptor activator of nuclear factor- $\kappa \mathrm{B}$ ligand; $\mathrm{I} \kappa \mathrm{B} \alpha$, inhibitor of $\kappa \mathrm{B} \alpha$; MAPK, mitogen-activated protein kinase.

from ZOL to denosumab markedly increased the risk of developing BRONJ in patients with bone metastases (60). That is likely due to ZOL having high affinity for bone hydroxyapatite, thus leading to prolonged drug action and excessive toxic effects. In general, it remains unknown which treatment strategy exhibits improved value from economic and health perspectives. In particular, comprehensive guidelines claim that there is insufficient evidence for recommending one of these bone-targeting drugs over another for the treatment of metastatic bone disease (61). Therefore, the clinical utilities of these two drugs require further exploration.

Accumulating evidence has indicated that $\mathrm{ZOL}$ inhibits osteoclast differentiation in vitro by affecting various signalling pathways. For example, evidence has indicated that ZOL acts by inhibiting farnesyl pyrophosphate synthase in the HMGA-CoA reductase pathway, also known as the mevalonate pathway (62-64). In addition, certain previous studies have indicated that ZOL suppresses the noncanonical $\mathrm{Wnt} / \mathrm{Ca}^{2+} / \mathrm{calmodulin}$-dependent protein kinase II pathway $(65,66)$. Certain studies have also demonstrated that ZOL is involved in the RANKL/RANK pathway. Specifically,
Pan et al (67) revealed that ZOL inhibits recruitment and osteoclastogenesis by significantly reducing the protein expression of transmembrane RANKL. It was also found that it does not markedly affect $R A N K L$ gene expression in osteoblast-like cells. Thus, there are conflicting results regarding the effects of ZOL on RANKL. In addition, Kimachi et al (55) reported that ZOL hinders osteoclast differentiation by inhibiting RANK expression and the migration of RAW264.7 and bone marrow cells; it was hypothesized that the inhibitory effects of ZOL on RANK expression may be associated with the inhibition of the NF-KB pathway. Cheng et al (68) further confirmed that ZOL modulates osteoclast apoptosis through activation of the NF- $\kappa \mathrm{B}$ signalling pathway using an ovariectomised rat model. Furthermore, ZOL has been reported to inhibit the NF- $\mathrm{KB}$ pathway by promoting the deubiquitination of TRAF6 (19). Our previous study also reported that ZOL reduces the levels of p-IкB $\alpha, p-p 65$ and p-JNK at different time points following RANKL exposure in RAW264.7 cells (32). In the present study, the expression levels of these signaling pathways were also found to be suppressed in a concentration-dependent manner. To the best of our knowledge, the present study also 
revealed for the first time that ZOL inhibits the nuclear translocation of p65 and the levels of the downstream factors c-Jun, c-Fos and NFATc1, thus decreasing the expression of the fusion-related molecule DC-STAMP and other osteoclast-specific markers in RAW264.7 cells. To a certain extent, the present data confirmed the previous conclusion that $\mathrm{NF}-\kappa \mathrm{B}$ and JNK signalling pathways may be involved in the inhibitory effects of ZOL on osteoclastogenesis.

However, the present study has several limitations. First, the effects of ZOL on other cell types, such as osteocytes and osteoblasts, need to be revealed through future investigations. Second, the findings of the present study need to be validated by additional assays. For example, luciferase activity assays could be used to further verify the activation of downstream factors; the findings could then be compared to establish their consistency with the present evidence. In addition, molecular docking assays could be performed to determine whether the expression of the gene encoding the osteoclast differentiation marker can be silenced by ZOL. In conclusion, the results of the present study demonstrated that ZOL suppressed both osteoclast formation and bone resorption in vitro. Mechanistically, the study confirmed that the inhibitory effects of ZOL occur via the inhibition of JNK and NF- $\mathrm{KB}$ activation, thus decreasing the downstream expression of c-Jun, c-Fos and NFATc1. This subsequently reduces the expression of the fusion-related molecule DC-STAMP, as well as other osteoclastic marker genes (Fig. 7). These results highlight the potential usefulness of ZOL in preventing osteoclast formation and provide further insights into the mechanism of action of ZOL in this context. Therefore, ZOL may be a potential target for the treatment of osteoclast-related diseases such as osteoporosis, and thus warrants further study.

\section{Acknowledgements}

All experiments were performed in the Key Laboratory of Endemic and Ethnic diseases of Guizhou Medical University (Guizhou, China).

\section{Funding}

The present study was supported by the Natural Science Foundation of China (grant nos. 81660179 and 82060207).

\section{Availability of data and materials}

The datasets used and/or analysed during the current study are available from the corresponding author on reasonable request.

\section{Authors' contributions}

JL and JPL conceived and designed the study; XLH and YTC performed the experiments; CL wrote the manuscript and designed the figures; and QZ and XMS analysed the data. XLH and JL confirm the authenticity of all the raw data. All authors read and approved the final manuscript.

\section{Ethics approval and consent to participate}

Not applicable.

\section{Patient consent for publication}

Not applicable.

\section{Competing interests}

The authors declare that they have no competing interests.

\section{References}

1. Li DZ, Zhang QX, Dong XX, Li HD and Ma X: Treatment with hydrogen molecules prevents RANKL-induced osteoclast differentiation associated with inhibition of ROS formation and inactivation of MAPK, AKT and NF-kappa B pathways in murine RAW264.7 cells. J Bone Miner Metab 32: 494-504, 2014.

2. Cosman F, de Beur SJ, LeBoff MS, Lewiecki EM, Tanner B, Randall S and Lindsay R; National Osteoporosis Foundation: Clinician's guide to prevention and treatment of osteoporosis. Osteoporos Int 25: 2359-2381, 2014.

3. Yang JH, Li B, Wu Q, Lv JG and Nie HY: Echinocystic acid inhibits RANKL-induced osteoclastogenesis by regulating NF- $\kappa \mathrm{B}$ and ERK signaling pathways. Biochem Biophys Res Commun 477: 673-677, 2016.

4. Madel MB, Ibáñez L, Wakkach A, de Vries TJ, Teti A, Apparailly F and Blin-Wakkach C: Immune function and diversity of osteoclasts in normal and pathological conditions. Front Immunol 10: 1408, 2019.

5. Islam R, Bae HS, Yoon WJ, Woo KM, Baek JH, Kim HH, Uchida T and Ryoo HM: Pin1 regulates osteoclast fusion through suppression of the master regulator of cell fusion DC-STAMP. J Cell Physiol 229: 2166-2174, 2014.

6. Lee JW, Kobayashi Y, Nakamichi Y, Udagawa N, Takahashi N, Im NK, Seo HJ, Jeon WB, Yonezawa T, Cha BY and Woo JT: Alisol-B, a novel phyto-steroid, suppresses the RANKL-induced osteoclast formation and prevents bone loss in mice. Biochem Pharmacol 80: 352-361, 2010.

7. Nakashima T and Takayanagi $\mathrm{H}$ : New regulation mechanisms of osteoclast differentiation. Ann NY Acad Sci 1240: E13-E18, 2011.

8. He Y, Zhang Q, Shen Y, Chen X, Zhou F and Peng D: Schisantherin A suppresses osteoclast formation and wear particle-induced osteolysis via modulating RANKL signaling pathways. Biochem Biophys Res Commun 449: 344-350, 2014.

9. Yen ML, Hsu PN, Liao HJ, Lee BH and Tsai HF: TRAF-6 dependent signaling pathway is essential for TNF-related apoptosis-inducing ligand (TRAIL) induces osteoclast differentiation. PLoS One 7: e38048, 2012.

10. Léotoing L, Wauquier F, Guicheux J, Miot-Noirault E, Wittrant $Y$ and Coxam V: The polyphenol fisetin protects bone by repressing NF- $\kappa \mathrm{B}$ and MKP-1-dependent signaling pathways in osteoclasts. PLoS One 8: e68388, 2013.

11. Cheng B, Li J, Du J, Lv X, Weng L and Ling C: Ginsenoside Rb1 inhibits osteoclastogenesis by modulating NF- $\kappa B$ and MAPKs pathways. Food Chem Toxicol 50: 1610-1615, 2012.

12. Soysa NS, Alles N, Aoki K and Ohya K: Osteoclast formation and differentiation: An overview. J Med Dent Sci 59: 65-74, 2012.

13. Lieben L: Bone: The concept of RANKL-independent osteoclastogenesis refuted. Nat Rev Rheumatol 12: 623, 2016.

14. Cai XJ, Wang Z, Cao JW, Ni JJ, Xu YY, Yao J, Xu H, Liu F and Yang GY: Anti-angiogenic and anti-tumor effects of metronomic use of novel liposomal zoledronic acid depletes tumor-associated macrophages in triple negative breast cancer. Oncotarget 8: 84248-84257, 2017.

15. Endo Y, Kumamoto H, Nakamura M, Sugawara S, TakanoYamamoto T, Sasaki K and Takahashi T: Underlying mechanisms and therapeutic strategies for bisphosphonate-related osteonecrosis of the jaw (BRONJ). Biol Pharm Bull 40: 739-750, 2017.

16. Elsayed R,Abraham P,Awad ME,KuragoZ,BaladhandayuthamB, Whitford GM, Pashley DH, McKenna CE and Elsalanty ME: Removal of matrix-bound zoledronate prevents post-extraction osteonecrosis of the jaw by rescuing osteoclast function. Bone 110: 141-149, 2018.

17. Russell RG: Bisphosphonates: The first 40 years. Bone 49: 2-19, 2011.

18. Qiao H, Wang TY, Yu ZF, Han XG, Liu XQ, Wang YG, Fan QM, Qin A and Tang TT: Structural simulation of adenosine phosphate via plumbagin and zoledronic acid competitively targets JNK/Erk to synergistically attenuate osteoclastogenesis in a breast cancer model. Cell Death Dis 7: e2094, 2016. 
19. Li X, Sun W, Li J, Wang M, Zhang H, Pei L, Boyce BF, Wang Z and Xing L: Clomipramine causes osteoporosis by promoting osteoclastogenesis via E3 ligase Itch, which is prevented by zoledronic acid. Sci Rep 7: 41358, 2017.

20. Li P, Yang H, Jia N, Jin X, Xu D and Shen Y: Experimental study on inhibitory effect of zoledronic acid on the action style of the osteoclast. Sheng Wu Yi Xue Gong Cheng Xue Za Zhi 34: 78-82, 2017 (In Chinese).

21. de Oliveira MA, Asahi DA, Silveira CAE, Lima LAPA, Glick M and Gallottini M: The effects of zoledronic acid and dexamethasone on osseointegration of endosseous implants: Histological and histomorphometrical evaluation in rats. Clin Oral Implants Res 26: e17-e21, 2015.

22. Weber JBB, Camilotti RS, Jasper J, Casagrande LCO and Maito FLDM: Effect of low-level laser therapy on tissue repair after dental extraction in rats administered zoledronic acid and dexamethasone. J Biomed Opt 22: 58001, 2017.

23. Allen MR, Chu TM and Ruggiero SL: Absence of exposed bone following dental extraction in beagle dogs treated with 9 months of high-dose zoledronic acid combined with dexamethasone. J Oral Maxillofac Surg 71: 1017-1026, 2013.

24. Subramanian G, Fritton JC, Iyer S and Quek SY: Atypical dental implant failure with long-term bisphosphonate treatment-akin to atypical fractures? Oral Surg Oral Med Oral Pathol Oral Radiol 114: e30-e35, 2012.

25. Qi M, Hu J, Li J, Li J, Dong W, Feng X and Yu J: Effect of zoledronate acid treatment on osseointegration and fixation of implants in autologous iliac bone grafts in ovariectomized rabbits. Bone 50: 119-127, 2012

26. Khosla S and Shane E: A crisis in the treatment of osteoporosis. J Bone Miner Res 31: 1485-1487, 2016.

27. Kim SC, Kim DH, Mogun H, Eddings W, Polinski JM, Franklin JM and Solomon DH: Impact of the U.S. food and drug administration's safety-related announcements on the use of bisphosphonates after hip fracture. J Bone Miner Res 31: 1536-1540, 2016

28. Nakagawa T, Ohta K, Uetsuki R, Kato H, Naruse T, Murodumi H, Yokoyama S, Sakuma M, Ono S and Takechi M: Zoledronate inhibits osteoclast differentiation via suppressing vascular endothelial growth factor receptor 2 expression. Biochem Genet 58 : 473-489, 2020

29. Dundar S, Yaman F, Gecor O, Cakmak O, Kirtay M, Yildirim TT, Karaman T and Benlidayi ME: Effects of local and systemic zoledronic acid application on titanium implant osseointegration: An experimental study conducted on two surface types. J Craniofac Surg 28: 935-938, 2017.

30. Chadha GK, Ahmadieh A, Kumar S and Sedghizadeh PP: Osseointegration of dental implants and osteonecrosis of the jaw in patients treated with bisphosphonate therapy: A systematic review. J Oral Implantol 39: 510-520, 2013

31. Kim HJ, Kim HJ, Choi Y, Bae MK, Hwang DS, Shin SH and Lee JY: Zoledronate enhances osteocyte-mediated osteoclast differentiation by IL-6/RANKL axis. Int J Mol Sci 2 : 1467, 2019.

32. Huang XL, Huang LY, Cheng YT, Li F, Zhou Q, Wu C, Shi QH, Guan ZZ, Liao J and Hong W: Zoledronic acid inhibits osteoclast differentiation and function through the regulation of NF- $\kappa \mathrm{B}$ and JNK signalling pathways. Int J Mol Med 44: 582-592, 2019

33. Chen G, Huang L, Wu X, Liu X, Xu Q, Li F, Dai M and Zhang B: Adiponectin inhibits osteoclastogenesis by suppressing NF- $\mathrm{KB}$ and $\mathrm{p} 38$ signaling pathways. Biochem Biophys Res Commun 503 2075-2082, 2018.

34. Jiao Z, Xu W, Zheng J, Shen P, Qin A, Zhang S and Yang C: Kaempferide prevents titanium particle induced osteolysis by suppressing JNK activation during osteoclast formation. Sci Rep 7: 16665, 2017.

35. Livak KJ and Schmittgen TD: Analysis of relative gene expression data using real-time quantitative PCR and the 2(-Delta Delta C(T)) method. Methods 25: 402-408, 2001

36. Shibata K, Yoshimura Y, Kikuiri T, Hasegawa T, Taniguchi Y, Deyama Y, Suzuki K and Iida J: Effect of the release from mechanical stress on osteoclastogenesis in RAW264.7 cells. Int J Mol Med 28: 73-79, 2011

37. Tsubaki M, Komai M, Itoh T, Imano M, Sakamoto $K$, Shimaoka H, Takeda T, Ogawa N, Mashimo K, Fujiwara D, et al: Nitrogen-containing bisphosphonates inhibit RANKL- and M-CSF-induced osteoclast formation through the inhibition of ERK1/2 and Akt activation. J Biomed Sci 21: 10, 2014.

38. Kang MR, Jo SA, Yoon YD, Park KH, Oh SJ, Yun J, Lee CW, Nam KH, Kim Y, Han SB, et al: Agelasine D suppresses RANKL-induced osteoclastogenesis via down-regulation of c-Fos, NFATc1 and NF-кB. Mar Drugs 12: 5643-5656, 2014.
39. Kong X, Wu W, Yang Y, Wan H, Li X, Zhong M, Zhao H, Su X, Jia S, Ju D and Lin N: Total saponin from anemone flaccida Fr. Schmidt abrogates osteoclast differentiation and bone resorption via the inhibition of RANKL-induced $N F-\kappa B$, JNK and p38 MAPKs activation. J Transl Med 13: 91, 2015.

40. Prideaux M, Findlay DM and Atkins GJ: Osteocytes: The master cells in bone remodelling. Curr Opin Pharmacol 28: 24-30, 2016.

41. Tseng HC,KanayamaK,KaurK,Park SH,ParkS, KozlowskaA,SunS, McKenna CE, Nishimura I and Jewett A: Bisphosphonate-induced differential modulation of immune cell function in gingiva and bone marrow in vivo: Role in osteoclast-mediated NK cell activation. Oncotarget 6: 20002-20025, 2015.

42. Otero JE, Chen T, Zhang K and Abu-Amer Y: Constitutively active canonical NF- $\kappa \mathrm{B}$ pathway induces severe bone loss in mice. PLoS One 7: e38694, 2012.

43. Kim HK, Kim MG and Leem KH: Osteogenic activity of collagen peptide via ERK/MAPK pathway mediated boosting of collagen synthesis and its therapeutic efficacy in osteoporotic bone by back-scattered electron imaging and microarchitecture analysis. Molecules 18: 15474-15489, 2013.

44. Sui X, Kong N, Ye L, Han W, Zhou J, Zhang Q, He C and Pan H: p38 and JNK MAPK pathways control the balance of apoptosis and autophagy in response to chemotherapeutic agents. Cancer Lett 344: 174-179, 2014

45. Zhai ZJ, Li HW, Liu GW, Qu XH, Tian B, Yan W, Lin Z, Tang TT, Qin A and Dai KR: Andrographolide suppresses RANKL-induced osteoclastogenesis in vitro and prevents inflammatory bone loss in vivo. Br J Pharmacol 171: 663-675, 2014.

46. Huh JE, Jung IT, Choi J, Baek YH, Lee JD, Park DS and Choi DY: The natural flavonoid galangin inhibits osteoclastic bone destruction and osteoclastogenesis by suppressing NF- $\kappa \mathrm{B}$ in collagen-induced arthritis and bone marrow-derived macrophages. Eur J Pharmacol 698: 57-66, 2013.

47. Yamanaka Y, Clohisy JC, Ito H, Matsuno T and Abu-Amer Y: Blockade of JNK and NFAT pathways attenuates orthopedic particle-stimulated osteoclastogenesis of human osteoclast precursors and murine calvarial osteolysis. J Orthop Res 31: 67-72, 2013

48. Park JH, Lee NK and Lee SY: Current understanding of RANK signaling in osteoclast differentiation and maturation. Mol Cells 40: 706-713, 2017.

49. Liu X, Qu X, Wu C, Zhai Z, Tian B, Li H, Ouyang Z, Xu X, Wang W, Fan Q, et al: The effect of enoxacin on osteoclastogenesis and reduction of titanium particle-induced osteolysis via suppression of JNK signaling pathway. Biomaterials 35: 5721-5730, 2014

50. Zhao Q, Wang X, Liu Y, He A and Jia R: NFATc1: Functions in osteoclasts. Int J Biochem Cell Biol 42: 576-579, 2010.

51. Lee JH, Jin H, Shim HE, Kim HN, Ha $\mathrm{H}$ and Lee $\mathrm{ZH}$ : Epigallocatechin-3-gallate inhibits osteoclastogenesis by down-regulating c-Fos expression and suppressing the nuclear factor-kappaB signal. Mol Pharmacol 77: 17-25, 2010.

52. Zhang C, Dou CE, Xu J and Dong S: DC-STAMP, the key fusion-mediating molecule in osteoclastogenesis. J Cell Physiol 229: 1330-1335, 2014.

53. Chiu YH, Mensah KA, Schwarz EM, Ju Y, Takahata M, Feng C, McMahon LA, Hicks DG, Panepento B, Keng PC and Ritchlin CT: Regulation of human osteoclast development by dendritic cell-specific transmembrane protein (DC-STAMP) J Bone Miner Res 27: 79-92, 2012.

54. Zeng XZ, He LG, Wang S, Wang K, Zhang YY, Tao L, Li XJ and Liu SW: Aconine inhibits RANKL-induced osteoclast differentiation in RAW264.7 cells by suppressing NF- $\kappa$ B and NFATc1 activation and DC-STAMP expression. Acta Pharmacol Sin 37: 255-263, 2016.

55. Kimachi K, Kajiya H, Nakayama S, Ikebe T and Okabe K: Zoledronic acid inhibits RANK expression and migration of osteoclast precursors during osteoclastogenesis. Naunyn Schmiedebergs Arch Pharmacol 383: 297-308, 2011

56. Lee H, Jin Y, Roh M, Tsacogianis TN, Park S, Choi NK and Kim SC: Risk of cataract surgery and age-related macular degeneration after initiation of denosumab vs zoledronic acid for osteoporosis: A multi-database cohort study. Drugs Aging 37: 311-320, 2020.

57. Kondo H, Okimoto N, Yoshioka T, Akahoshi S, Fuse Y, Ogawa T, Okazaki Y, Katae Y, Tsukamoto M, Yamanaka Y, et al: Zoledronic acid sequential therapy could avoid disadvantages due to the discontinuation of less than 3-year denosumab treatment. J Bone Miner Metab 38: 894-902, 2020. 
58. Mori T, Crandall CJ, Fujii T and Ganz DA: Cost-effectiveness of zoledronic acid compared with sequential denosumab/alendronate for older osteoporotic women in Japan. Arch Osteoporos 16: 113, 2021.

59. Ikesue H, Mouri M, Tomita H, Hirabatake M, Ikemura M, Muroi N, Yamamoto S, Takenobu T, Tomii K, Kawakita M, et al: Associated characteristics and treatment outcomes of medication-related osteonecrosis of the jaw in patients receiving denosumab or zoledronic acid for bone metastases. Support Care Cancer 29: 4763-4772, 2021.

60. Ikesue H, Doi K, Morimoto M, Hirabatake M, Muroi N, Yamamoto S, Takenobu T and Hashida T: Switching from zoledronic acid to denosumab increases the risk for developing medication-related osteonecrosis of the jaw in patients with bone metastases. Cancer Chemother Pharmacol 87: 871-877, 2021

61. Chen C, Li R, Yang T, Ma L, Zhou S, Li M, Zhou Y and Cui Y: Denosumab versus zoledronic acid in the prevention of skeletal-related events in vulnerable cancer patients: A meta-analysis of randomized, controlled trials. Clin Ther 42: 1494-1507.e1, 2020.

62. Yang $G$, Singh $S$, Chen Y, Hamadeh IS, Langaee $T$, McDonough CW, Holliday LS, Lamba JK, Moreb JS, Katz J and Gong Y: Pharmacogenomics of osteonecrosis of the jaw. Bone 124: 75-82, 2019.

63. Fliefel RM, Entekhabi SA, Ehrenfeld $M$ and Otto S: Geranylgeraniol $(\mathrm{GGOH})$ as a mevalonate pathway activator in the rescue of bone cells treated with zoledronic acid: An in vitro study. Stem Cells Int 2019: 4351327, 2019.
64. Nakagawa T, Ohta K, Kubozono K, Ishida Y, Naruse T, Takechi M and Kamata N: Zoledronate inhibits receptor activator of nuclear factor kappa-B ligand-induced osteoclast differentiation via suppression of expression of nuclear factor of activated T-cell $\mathrm{cl}$ and carbonic anhydrase 2. Arch Oral Biol 60: 557-565, 2015.

65. Cui P, Liu H, Sun J, Amizuka N, Sun Q and Li M: Zoledronate promotes bone formation by blocking osteocyte-osteoblast communication during bone defect healing. Histol Histopathol 33: 89-99, 2018.

66. Zhang J, Park J, Lee JW, Kwon YD and Kim EC: Bisphosphonates hinder osteoblastic/osteoclastic differentiation in the maxillary sinus mucosa-derived stem cells. Clin Oral Investig 22: 1933-1943, 2018

67. Pan B, Farrugia AN, To LB, Findlay DM, Green J, Lynch K and Zannettino AC: The nitrogen-containing bisphosphonate, zoledronic acid, influences RANKL expression in human osteoblast-like cells by activating TNF-alpha converting enzyme (TACE). J Bone Miner Res 19: 147-154, 2004.

68. Cheng YT, Liao J, Zhou Q, Huo H, Zellmer L, Tang ZL, Ma H, Hong W and Liao DJ: Zoledronic acid modulates osteoclast apoptosis through activation of the NF- $\mathrm{kB}$ signaling pathway in ovariectomized rats. Exp Biol Med (Maywood) 246: 1727-1739, 2021. International (CC BY-NC-ND 4.0) License. 